\title{
Les parties prenantes de la gestion du risque de crue
}

\author{
The stake holders of the flood risk management
}

\author{
par P.H. Bourrelier \\ Conseil Général des Mines
}

Diverse stake holders must participate in the flood risk management, which consists in country planning, medium maintenance and supervision/alarm devices. A good cooperation between the different stake holders is necessary.

\section{LES PRINCIPES D'UNE GESTION PARTICIPATIVE}

\subsection{Principe participatif de la gestion des risques}

Les risques, tels que les risques de crue, qui sont récurrents, s'inscrivent sur une boucle : crise, réparation, prévention : la prévention est antérieure à la réalisation du risque, mais elle prépare ainsi la gestion de crise et doit être un des éléments des actions de la réparation post accidentelle; les décisions importantes se prennent à ce moment, et c'est ainsi que se constitue une appréciation du déroulement des événements (retour d'expérience) et que la mémoire se structure.

Le principe fondamental de la prévention est qu'elle doit faire participer toutes les "parties prenantes" au risque ; les Américains les désignent par le terme : "stake holder", c'està-dire littéralement ceux qui détiennent les enjeux : les sociologues emploient couramment en France le terme d'acteurs (ceux qui agissent).

Nous allons, dans la suite de l'exposé, examiner quelles sont ces parties et comment elles peuvent être impliquées.

1.2 Principe de continuité : situations courantes, situations aléatoires, situations extrêmes

La gestion d'une rivière et la vie autour d'elle doivent se concevoir en considérant l'état hydrologique courant, mais doivent aussi prendre en considération les situations exceptionnelles de sécheresse et de crue ; la variabilité fait partie de la nature, et jusqu'à un certain niveau, les crues sont des événements utiles ; au-delà, elles mettent à l'épreuve les dispositifs de prévention ; les événements extrêmes peuvent faire échec à certains d'entre eux, et il importe de prendre en considération cette éventualité, bien que sa très faible probabilité rende difficile son appréciation, c'est-à-dire la construction des scénarios.
Les acteurs de la gestion des risques comprennent des intervenants spécialisés dans les opérations de secours, de réparation et de prévention, en vertu du principe précédent. mais aussi toutes les parties prenantes de la gestion de la rivière et de l'eau en situation normale.

\section{II $\square$ COMPOSANTS TECHNIQUES DE LA GESTION DES RISQUES}

Pour mieux saisir les implications de ces principes, il y a lieu d'examiner quelle est l'intervention spécifique des acteurs dans chacune des phases.

\subsection{Prévention}

La prévention comporte elle-même trois axes :

a) occupation du sol et aménagement du territoire ; dispositifs de "mitigation",

b)entretien du milieu (lit, berges, bassins versants) et ouvrages de protection,

c) dispositifs de surveillance et d'alerte.

A des titres divers, ces catégories d'actions concernent, comme parties prenantes :

- les maires, les municipalités et leurs services techniques, - les riverains, les syndicats d'aménagements, les entités interdépartementales, les Agences de l'Eau,

- des concessionnaires et autres intervenant sur les cours d'eau, ou susceptibles de modifier l'écoulement,

- des services et entités de l'Etat qui ont des fonctions d'information, d'orientation et d'assistance ou de contrôle de légalité,

- la population, à titre d'occupant du sol soumis aux effets du risque. 


\subsection{Gestion de crise}

Interviennent en outre les services de secours et, le cas échéant, le préfet.

\subsection{Réparation}

Interviennent en outre les assureurs, et d'autres acteurs financiers.

\section{III — LA GESTION INTÉGRÉE ; L'EXPER- TISE ET L'INFORMATION}

Une gestion efficace suppose une participation intégrée de tous ces acteurs, dans toutes les phases, avec une définition aussi claire que possible de leurs compétences respectives.

L'acquisition et la diffusion des connaissances, bases de l'expertise, constituent un socle commun. Ce sont donc des volets essentiels de la gestion des risques et des processus de prise de décision, de sorte que les centres de compétence (organismes scientifiques, sociétés savantes) doivent aussi être considérés comme parties prenantes.

\section{IV $\square$ LES ÉCHELLES PERTINENTES}

Le dernier principe est celui qui consiste à organiser les actions et les coopérations aux échelles pertinentes, avec une bonne articulation d'une échelle à l'autre :

- échelle mondiale et continentale : surveillance et modélisation météorologique mondiale, action sur le climat, coopération pour les secours exceptionnels,

- échelle nationale : principes normatifs et coordination de la prévention, des secours et de la réparation,
- bassins fluviaux qui peuvent être d'échelle soit supranationale (ex : Rhin, Nil), soit infranationale,

- sous-bassins, sections des rivières : idem à cette échelle,

- communes : idem à cette échelle, avec un accent sur la communication avec la population.

\section{V — CONCLUSION, PROBLÈMES PARTICULIERS}

L'évaluation de la politique publique française a fait apparaître que la coopération entre tous ces intervenants dans le cadre d'une bonne répartition des compétences demanderait à la fois un effort de clarification et une volonté d'implication : - clarification : l'impératif essentiel est la clarification entre les fonctions de l'Etat et celles des collectivités territoriales à différents niveaux et en fonction des échelles.

- implication : les participations ou implications suivantes sont apparues fondamentales et actuellement insuffisantes :

- celle des départements et d'établissements intercommunaux,

- celle des Agences de bassins qui doivent apporter une contribution au titre des connaissances et de la mise en place des transactions entre intervenants.

- celle des entreprises concessionnaires, délégataires de service public,...

- celle des assureurs qui doivent participer à la connaissance et à l'expertise (retour d'expérience, signaux sur les niveaux des risques), et inciter à des réparations incluant des efforts de prévention,

- en définitive, et de façon incontournable, la population et ses associations. 